\title{
Video Article \\ Measurements of Long-range Electronic Correlations During Femtosecond Diffraction Experiments Performed on Nanocrystals of Buckminsterfullerene
}

Rebecca A. Ryan ${ }^{1}$, Sophie Williams ${ }^{1}$, Andrew V. Martin ${ }^{1}$, Ruben A. Dilanian ${ }^{1}$, Connie Darmanin ${ }^{2}$, Corey T. Putkunz ${ }^{1}$, David Wood ${ }^{3}$, Victor A. Streltsov ${ }^{4}$, Michael W.M. Jones ${ }^{5}$, Naylyn Gaffney ${ }^{6}$, Felix Hofmann ${ }^{7}$, Garth J. Williams ${ }^{8}$, Sebastien Boutet ${ }^{9}$, Marc Messerschmidt ${ }^{10}$, M. Marvin Seibert ${ }^{11}$, Evan K. Curwood ${ }^{11}$, Eugeniu Balaur ${ }^{2}$, Andrew G. Peele ${ }^{5}$, Keith A. Nugent ${ }^{2}$, Harry M. Quiney ${ }^{1}$, Brian Abbey ${ }^{2}$

${ }^{1}$ ARC Centre of Excellence in Advanced Molecular Imaging, School of Physics, University of Melbourne

${ }^{2}$ Australian Research Council (ARC) Centre of Excellence in Advanced Molecular Imaging, Department of Chemistry and Physics, La Trobe Institute for Molecular Sciences, La Trobe University

${ }^{3}$ Department of Physics, Imperial College London

${ }^{4}$ Florey Institute of Neuroscience and Mental Health

${ }^{5}$ Science and Engineering Faculty, Queensland University of Technology

${ }^{6}$ Swinburne University of Technology

${ }^{7}$ Department of Engineering Science, University of Oxford

${ }^{8}$ Brookhaven National Laboratory

${ }^{9}$ Linac Coherent Light Source, SLAC National Accelerator Laboratory

${ }^{10}$ BioXFEL Science and Technology Center

${ }^{11}$ Laboratory of Molecular Biophysics, Department of Cell and Molecular Biology, Uppsala University

${ }^{12}$ Australian Synchrotron

Correspondence to: Harry M. Quiney at quiney@unimelb.edu.au, Brian Abbey at B.Abbey@latrobe.edu.au

URL: https://www.jove.com/video/56296

DOI: doi:10.3791/56296

Keywords: Chemistry, Issue 126, Nanocrystallography, Femtosecond X-ray Diffraction, Correlated Electron Dynamics, X-ray Free Electron Lasers, Linac Coherent Light Source, Buckminsterfullerene

Date Published: 8/22/2017

Citation: Ryan, R.A., Williams, S., Martin, A.V., Dilanian, R.A., Darmanin, C., Putkunz, C.T., Wood, D., Streltsov, V.A., Jones, M.W., Gaffney, N., Hofmann, F., Williams, G.J., Boutet, S., Messerschmidt, M., Seibert, M.M., Curwood, E.K., Balaur, E., Peele, A.G., Nugent, K.A., Quiney, H.M., Abbey, B. Measurements of Long-range Electronic Correlations During Femtosecond Diffraction Experiments Performed on Nanocrystals of Buckminsterfullerene. J. Vis. Exp. (126), e56296, doi:10.3791/56296 (2017).

\section{Abstract}

The precise details of the interaction of intense X-ray pulses with matter are a topic of intense interest to researchers attempting to interpret the results of femtosecond X-ray free electron laser (XFEL) experiments. An increasing number of experimental observations have shown that although nuclear motion can be negligible, given a short enough incident pulse duration, electronic motion cannot be ignored. The current and widely accepted models assume that although electrons undergo dynamics driven by interaction with the pulse, their motion could largely be considered 'random'. This would then allow the supposedly incoherent contribution from the electronic motion to be treated as a continuous background signal and thus ignored. The original aim of our experiment was to precisely measure the change in intensity of individual Bragg peaks, due to X-ray induced electronic damage in a model system, crystalline $\mathrm{C}_{60}$. Contrary to this expectation, we observed that at the highest $\mathrm{X}$-ray intensities, the electron dynamics in $\mathrm{C}_{60}$ were in fact highly correlated, and over sufficiently long distances that the positions of the Bragg reflections are significantly altered. This paper describes in detail the methods and protocols used for these experiments, which were conducted both at the Linac Coherent Light Source (LCLS) and the Australian Synchrotron (AS) as well as the crystallographic approaches used to analyse the data.

\section{Video Link}

The video component of this article can be found at https://www.jove.com/video/56296/

\section{Introduction}

One of the major purposes of X-ray free electron lasers (XFELs) is to develop a high throughput, high resolution approach to molecular imaging and dynamics. Structural biology depends on atomic scale information, traditionally limited to lower resolution X-ray crystallography techniques performed at third generation synchrotrons. Long exposure times which cause significant radiation damage in crystals, greatly affect the resolution achieved using traditional techniques. The snapshot diffraction imaging scheme ${ }^{2,3,4}$ employed at XFELs, involves collecting diffraction images from short pulse X-rays hitting either fixed target samples (which are translated across the beam focus) or samples injected into the path of the beam. 
The XFEL pulse-sample interaction ultimately destroys the samples, due to the onset of severe radiation damage. The diffraction images are collected before the onset of this destruction due to the sub-100 fs pulse durations. The ability to determine high resolution structures from nanocrystals is rapidly becoming well established. However, dynamical processes which occur on femtosecond timescales under experimental imaging conditions offer deeper insights into atomic physics and can have a macroscopic effect on nanocrystals and their diffraction patterns ${ }^{5,6,7}$.

Whilst catastrophic structural damage is avoided on the femtosecond timescale during which a snapshot diffraction image is recorded, the power density of an XFEL pulse may be high enough to modify the electronic properties of the sample with which the X-rays interact ${ }^{7,8,9}$. An exploration of the physics of the interaction of intense coherent X-ray pulses with matter is not only of intrinsic scientific interest, but will be critically important to the interpretation of any experiment in which light from an XFEL pulse is used to explore structure.

In X-ray imaging experiments performed on single molecules, small clusters, or nanocrystals comprised of a few unit cells, perturbative analysis indicates that one should observe both a reduction in the apparent coherence of the scattered signal ${ }^{8}$, and the growth of a structureless background signal as a result of electrodynamical processes ${ }^{9}$. This experiment sought to assess the degree to which decoherence due to electrodynamical processes, occurs in powdered nanocrystalline $\mathrm{C}_{60}$ due to interaction with short XFEL pulses.

In this article, we provide details regarding the experimental procedure in which a highly ordered transient electronic structure from $\mathrm{C}_{60}$ nanocrystals is observed due to interaction with an XFEL pulse ${ }^{1}$. The diffraction pattern produced under these conditions is significantly different from that observed when the same sample is illuminated by lower power, but otherwise identical XFEL pulses, or when a synchrotron beam at the same photon energy is used. This difference is marked by the presence of Bragg peaks that are not seen in the two diffraction profiles corresponding to the low-power and synchrotron diffraction images. We demonstrate our analysis and model-fitting approach, used to confirm the presence of a dynamic electronic distortion induced by XFEL pulse-nanocrystal interaction.

\section{1. $\mathrm{C}_{60}$ Powder Sample Preparation}

1. Apply polyamide film, $10 \mu \mathrm{m}$ thick, to one side of the fixed $1 \mathrm{~mm}$ thick aluminium sample holder (design shown in Figure 1a).

2. Crush the $\mathrm{C}_{60}$ using a pestle and mortar in small batches of approximately $100 \mu \mathrm{g}$. The amount of sample added to the mortar is not critical, but ensure that it does not exceed the height of the rounded end of the pestle so that you don't risk compacting the powder instead of crushing it. This will ensure that fine nanocrystals are produced. Repeat this process several times to obtain the amount required for data collection.

CAUTION: Preparation and handling of nanomaterials should only be performed within biosafety cabinets.

3. Remove the crushed $\mathrm{C}_{60}$ powder directly from the mortar using a small spatula and spread as thinly as possible across the cells of the sample holder, with the adhesive side of the polyimide film backing in the sample holder facing the powder.

1. To successfully create a uniform monolayer, place the adhesive side of a second polyimide film (also $10 \mu \mathrm{m}$ thick) directly over the powder in the sample holder and pull off. Excess $C_{60}$ powder will stick to the second piece of tape and can then be removed from the sample holder.

2. Repeat until no more powder comes off the tape and a monolayer of $\mathrm{C}_{60}$ powder appears evenly spread across individual sample holder cells (see Figure 1b).

4. Leave at least one window blank (no sample) to enable a polyimide background to be recorded.

5. Seal the sample holder in a plastic container for transport to the beamline sample chamber.

\section{Preliminary Australian Synchrotron Studies}

1. Use $\mathrm{C}_{60}$ prepared as in Steps $1.1-1.5$ for powder diffraction at the MX2 beamline at the Australian Synchrotron.

2. Request an incident energy of $12.905 \mathrm{keV}(0.9607 \AA)$ and a beam size of $30 \times 7 \mathrm{um}$.

3. Mount the $\mathrm{C}_{60}$ sample holder on a standard crystallography pin, vertical to the X-ray beam on the goniometer at a distance of around $625 \mathrm{~mm}$ from the detector, to enable high-resolution data to be collected.

4. Move the cryogenic nozzle out of position to ensure room temperature powder diffraction data is collected.

5. Open the Blu-Ice ${ }^{10}$ software program which controls the MX2 beamline.

6. Press the 'Start' button to begin data collection.

7. Test different exposure times (by repeating steps 2.5 and 2.6) to ensure sufficient powder data is collected in the image (characterised by strong diffraction rings out to the edge of the detector) maximising the dynamic range of the signal without saturating the detector.

8. Scan the $\mathrm{C}_{60}$ sample window and collect multiple images from the same sample.

\section{Request XFEL Parameters for Beamline Setup}

1. Request the shortest possible pulse duration available at the LCLS (April 2012 - 32 fs FWHM), without significant loss of flux ('high-charge mode') for the experiment at the LCLS Coherent X-ray Imaging (CXI) ${ }^{11}$ beamline.

2. Request the use of $10 \mathrm{keV}(1.24 \AA)$ incident X-ray energy.

3. Request the smallest focal spot size practically achievable using CXI Kirkpatrick-Baez (KB) mirrors on the order of $100 \times 100 \mathrm{~nm}^{2}$. NOTE: Adjusting the pulse duration and beam focussing is performed by the scientists at the CXI beamline. The unfocused beam size upstream of the KB optics was approximately $800 \times 800 \mu \mathrm{m}^{2}$, after focusing the beam size achieved at the focal spot was approximately 300 $\times 300 \mathrm{~nm}^{2} \mathrm{FWHM}$, as determined by optical microscopy of craters made by the XFEL beam in YAG crystals.

4. Request a sample - detector distance (Z-distance) of $79 \mathrm{~mm}$ (Figure 1c). 


\section{Record the Darkfield}

1. Set the data recording parameters in the DAQ (Data Acquisition) ${ }^{12}$ control panel: beam (off), number of events (500), event recording device (Cornell-SLAC Pixel Array Detector - CSPAD13).

2. Press 'Record Run' when ready to record a dataset of snapshot images. NOTE: The dataset of all events recorded is called a 'run' and is saved in the .XTC file format.

\section{Record the $10 \%$ Incident XFEL Flux Run}

1. Request the placement of appropriate thickness of aluminium attenuator downstream of the sample, and directly in front of the Cornell-SLAC Pixel Array Detector (CSPAD) ${ }^{13}$ in order to protect it from damage.

2. Request the insertion of silicon attenuators upstream of the sample at a thickness calculated to attenuate $90 \%$ of the incident $X$-rays hitting the sample. Note that the pulse flux is estimated from the beam current monitor. The fluence at the sample was estimated to be $8.3 \times 10^{1 \%}$ photons $/ \mathrm{mm}^{2} /$ pulse.

3. Mount the sample holder containing the $\mathrm{C}_{60}$ sample in the $\mathrm{CXI}$ vacuum chamber.

4. Request the beamline scientists to perform the vacuum pump procedure for the sample chamber until vacuum is reached. It takes around 30 minutes to reach $10^{-7}$ Tor, at room temperature.

5. Set the data recording parameters in the DAQ control panel: beam (on), events (1500), event recording device (CSPAD).

6. Click the 'Scan' button in the configuration section of the DAQ GUI window.

7. Provide parameters to the beamline scientists to set the raster scan procedure for the run. These include the start position (top left corner) and end position (lower right corner) of one sample holder cell window, the step sizes $(600 \mu \mathrm{m})$ and the direction of the scan movement (in the x-axis). Using these parameters, one cell window (shown in Figure 1 a) permits twenty scans in the x-direction over three rows of the cell window.

8. Press 'Apply' when the correct values have been entered.

9. Request the beamline scientists to set the pulse repetition rate to $1 \mathrm{~Hz}$. Note: The available pulse repetition rate at the LCLS is $120 \mathrm{~Hz}$, however in the fixed-target scanning mode ${ }^{14}$, a lower pulse repetition rate is required to avoid the possibility of measuring sample that has already been damaged by a previous shot. CAUTION: XFEL interaction with the aluminium sample frame poses the risk of saturation and damage of the CSPAD and so care must be taken to avoid the frame.

10. Press 'Record Run' to record an XFEL snapshot powder diffraction dataset.

11. Using the computing environment available at LCLS ${ }^{12}$, navigate to the file directory that the data is recorded in within in a terminal window.

12. Type the command 'xtcexplorer/filepath/filename' to open the XTC file explorer GUI and view images recorded in the run.

13. Check images for possible detector saturation, which typically occurs at around 1,4000 ADUs $^{13}$. If the detector shows any signs of saturation the aluminium attenuation at the detector needs to be increased. If this occurs, request more layers of aluminium attenuator at the detector and repeat steps 5.6 - 5.12 with the raster scan set for the next sample holder cell window. A layer of aluminium $100 \mu \mathrm{m}$ thick, covering the central four modules of the CSPAD was used for this dataset.

\section{Record the $100 \%$ XFEL Flux Run}

1. Request the insertion of thick aluminium attenuator $(1,000 \mu \mathrm{m})$ over the central four modules of the CSPAD and thinner $100 \mu \mathrm{m}$ thick attenuator over the outer four modules.

2. Request the removal of silicon attenuators to allow $100 \%$ of the available $X$-ray flux to hit the sample. The peak unattenuated incident peak flux was estimated to be $7.5 \times 10^{11}$ photons /pulse (giving a fluence of approximately $8.3 \times 1018$ photons $/ \mathrm{mm} 2 /$ pulse at the sample).

3. Repeat steps $5.5-5.12$ to record the diffraction dataset on a new sample holder cell window.

4. Repeat step 5.13 to monitor detector saturation status and determine whether sufficient Bragg powder diffraction rings or Bragg spots (out to the edge of the detector) are visible and well defined.

\section{XFEL Data Post-processing and Peak Analysis}

1. Retrieve the calibration file (or file path) from the beamline scientists.

NOTE: The CSPAD data in a dataset is provided as individual detector panels grouped by an event number (corresponding to one image frame snapshot). The calibration file is needed to assemble detector panels into the correct relative positions to produce a reconstructed image frame corresponding to the whole detector.

2. Extract darkfield frames (example shown in Figure 2a) from the darkfield run dataset using the python scripting language and applying the calibration file. Note: software and an established data processing pipeline for serial femtosecond crystallography experiment at XFELS which was not available at the time of this experiment is now available ${ }^{15,16}$.

3. Sum the darkfield frames and generate an average darkfield image. Save this as the darkfield.

4. Extract the diffraction frame images from the diffraction run datasets (an example quadrant is shown in Figure 2b), and apply a darkfield subtraction. The sparsity of the signal produced in individual frames (after darkfield and background correction) is shown in Figure 2c.

5. Sum the darkfield corrected diffraction images to produce the final $2 \mathrm{D}$ powder diffraction image (Figure 2d).

6. Load the powder diffraction image as the input file in FIT2D ${ }^{17}$ (a GUI data reduction program).

7. Enter the dimensions of the image (x-length and y-length as 1,800 pixels) and select 'POWDER DIFFRACTION (2D) '.

8. Click 'Beam Centre' to locate the center of the diffraction rings. Select four points on the inner most diffraction ring (approximately equally spaced). Press 'CONTINUE' to determine the center of the diffraction pattern array.

9. Click 'Integrate' to perform an azimuthal integration of the diffraction image.

10. Enter the geometry parameters: pixel size (110 microns), sample-detector distance $(79 \mathrm{~mm})$, wavelength (1.24 Angstrom) and press continue to generate a 1D powder diffraction pattern.

11. Export the powder diffraction pattern as a .chi file to produce an array of scattering angle $(2 \theta)$ versus intensity values. 
12. Determine the background represented by the scattering from the polyimide polyamide backing on the sample using appropriate software. Note: The authors in this experiment used Powder ${ }^{18}$ and RIETAN ${ }^{19}$ to perform background subtraction from the 1D powder diffraction pattern.

13. Perform steps 7.1 - 7.9 for the dataset runs recorded for the different XFEL intensities.

14. Select the highest intensity value out of the three powder diffraction profiles.

15. Normalize all of the profiles to the most intense peak in the pattern - the (111) peak

16. Plot the 1D X-ray powder diffraction patterns obtained from the Australian Synchrotron (described in Protocol section 2), the 100\% flux case and the $10 \%$ flux case on the same axes using generic plotting software (Figure 3 a-c).

17. Optional step: Characterize the structure by performing additional analysis methods of your choice. Crystallographic data analysis from this experiment was performed using the program RIETAN-2000 (incorporating the split pseudo-Voigt function of Toraya ${ }^{20,21}$ as a profile function) to analyse Bragg reflections. Maximum Entropy Analysis was performed using the software PRIMA ${ }^{22}$ to confirm that the structure relating to the $10 \%$ XFEL intensity and Australian Synchrotron datasets matched the published structure for room temperature $F C C \mathrm{C}_{60}$.

\section{Representative Results}

\section{XFEL Powder Diffraction}

The data presented for the $100 \%$ incident flux XFEL powder diffraction is the result of summing more than 1000 single-shot measurements to produce a complete powder-ring with a resolution of better than $2 \AA$.

\section{Powder diffraction profiles comparison}

The Bragg peaks for the diffraction rings were identified and scaled to the first (most intense) peak reflection (111). Figure 3 shows the three different diffraction line profiles. By comparing the line profiles of the three diffraction patterns, we observe that the diffraction data recorded at the Australian Synchrotron is almost identical to the Bragg profile seen in the $10 \%$ XFEL data. Some very minor differences in the relative heights of the Bragg peaks, but not their positions are observed. In stark contrast, the profile of the $100 \%$ power XFEL powder diffraction data reveals the presence of additional peaks not seen in the 10\% XFEL data profile, nor in the Synchrotron data profile. The locations of these extra reflections are identified in Table 1. In order to interpret these differences, an adjustment to the model of expected diffraction from a room temperature FCC $\mathrm{C}_{60}$ crystal was constructed.

\section{X-ray diffraction modelling of the room temperature FCC $\mathrm{C}_{60}$ structure}

The intensity of powder diffraction peaks associated with Bragg reflections from a crystal is given by

$I \vec{Q})=K \mu L_{p}\left|\Phi(Q) \sum_{m}^{M} \exp \left(-2 \pi i \vec{Q} \cdot \overrightarrow{r_{m}}\right)\right|^{2} W(\vec{Q})$

where $\vec{Q}$ is the scattering vector, $K$ is the scale factor, $\mu$ is the multiplicity factor, $L_{\mathrm{p}}$ is the Lorentz-polarization factor, $W(\vec{Q})$ is the peak profile function and $M$ is the number of $C_{60}$ molecules contained in the scattering volume located at positions $r_{\mathrm{m}}$. The molecular form factor (MFF), $\Phi(Q)$, for a $\mathrm{C}_{60}$ molecule is given by

$\Phi(\vec{Q})=f_{c}(\vec{Q}) \sum_{j=1}^{60} \exp \left(2 \pi i \vec{Q} \cdot \overrightarrow{r_{j}}\right)$

where $r_{j}$ is the position of the $j^{\text {th }}$ carbon atom in the molecule and $f_{c}$ is the atomic scattering factor of the carbon atom.

The unit cell parameters of the crystal define the positions of allowed reflections for an X-ray powder diffraction pattern. Using the known room temperature FCC parameters (unit cell length, molecule positions within the unit cell) of $\mathrm{C}_{60}$, together with the experimental geometry in the $\mathrm{X}$-ray diffraction experiment, the expected positions of peaks (Bragg reflections) can be calculated using the MFF for $\mathrm{C}_{60}$ and Eq. 1 and Eq. 2 .

\section{X-ray diffraction modelling of $100 \%$ XFEL data}

We begin by assuming that significant distortions/transformations or displacements of the nuclei from their ideal positions do not occur during the $32 \mathrm{fs}$ duration of the incident pulse as suggested in prior studies ${ }^{23,24}$. Rather, that significant change in the intensities seen in the $100 \%$ XFEL data must instead be driven by motions of the electronic structure of the $\mathrm{C}_{60}$ molecules. In the following we describe a model that reproduces the experimentally observed features of the $100 \%$ XFEL diffraction data, via a modification of the centro-symmetric distribution of the $\mathrm{C}_{60}$ molecules.

In its normal, neutral state, the crystalline structure of $\mathrm{C}_{60}$ is maintained by dipolar forces that are induced by instantaneous fluctuations in its electron density. Under the experimental conditions described here, however, the ionization of the system generates a strong internal electric field that induces electric dipole moments in the molecules by polarization. Previously the formation of dipoles in $\mathrm{C}_{60}$ has only been observed in single molecules and small clusters using optical techniques such as UV spectroscopy ${ }^{25}$. Here however, the redistribution of the electron density observed is evidently both long-range and long-lived relative to the duration of the XFEL pulse so that its effects are observed in the crystallographic X-ray diffraction pattern.

This results in the alignment of neighbouring dipoles via a Coulomb interaction, and a decoupling of the electronic structure from the underlying

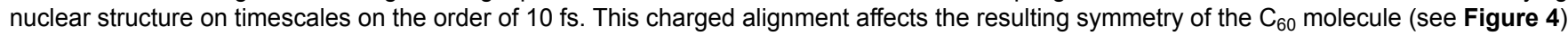
The loss of the spherical symmetry of the molecule leads to an additional phase contribution to the scattering amplitude, since the MFFs of $\mathrm{C}_{60}$ molecules are no longer real but complex functions. 
A periodically varying MFF was used to model the occurrence of an asymmetric molecular charge distribution in which the distribution of the electron density of the $m^{\text {th }}$ molecule is displaced relative to its position in the crystal structure. With this modification to the $\mathrm{C}_{60}$ MFF, we were able to replicate the intensity profile seen in the $100 \%$ XFEL data.

Eq. 2 provides the basis for constructing an expression for the scattering factor, which captures the long-range electronic correlations formed from the XFEL-induced dipoles in the $100 \%$ XFEL data. From this a new MFF function, modified to account for the polarized $\mathrm{C}_{60}$ molecules, can be constructed:

$\Phi^{\prime}(\vec{Q}, \delta \vec{r})=\Phi(\vec{Q}) \exp (i 2 \pi \vec{Q} \cdot \delta \vec{r})$

where $\Phi(\vec{Q})$ is the MFF of the ideal $\mathrm{C}_{60}$ molecules (given by Eq. 2) and $\delta \overrightarrow{\boldsymbol{r}}$ defines the polarization vector of the XFEL induced dipole. In the limit $|\delta \vec{r}|=0$, Eq. 3 approximates Eq. 2, and the room temperature 10\% power diffraction data is recovered. As $|\delta \vec{r}|$ increases, the symmetry of the molecule is altered, and the ratios of all the possible diffraction peaks begin to vary. The actual distribution of polarized molecules in a cubic lattice affects the resulting diffraction pattern.

When $|\delta \vec{r}|>0$, the symmetry of the $\mathrm{C}_{60}$ molecule is altered and the ratios of all the possible diffraction peaks begin to vary relative to the low-power diffraction pattern. To fit the data to this model, values of $0 \leq|\delta \vec{r}| \leq 1$ were explored, showing good agreement in the $20^{\circ} \leq 2 \theta \leq$ $30^{\circ}$ range of scattering angles for $|\delta \vec{r}|=0.75 \AA$.

The intended purpose of this experiment was to measure the degree to which stochastic photoionisation of the K-shell in carbon atoms affects the diffracted intensities measured for $\mathrm{FCC} \mathrm{C}_{60}$ nanocrystals. Photoionisation of the K-shell electrons in carbon atoms (electron binding energy $=$ $284 \mathrm{eV}$ ) modifies the atomic scattering factors, $f_{c}$, seen as a reduced scattering amplitude within the high $\vec{Q}$ scattering regions. K-shell holes in carbon atoms within $\mathrm{C}_{60}$ molecules arranged in a crystalline lattice causes modifications of the scattering amplitudes of the Bragg reflections.

We expected to observe a growing isotropic background, dependent on the photon flux applied to powdered nanocrystal samples according to the following fundamental assumptions: 1) that the photoionisation of the K-shell in carbon is the dominant process in the sample-XFEL interaction, 2) that photoionisation of individual carbon atoms is not correlated to any other atoms in the crystal, 3) that photoionized electrons remain delocalized for the duration of the pulse and hence contribute to the continuous background signal.

What we actually observed in the experiment was the presence of strong, forbidden reflections in room temperature, $\mathrm{FCC}$ nanocrystals of $\mathrm{C}_{60}$ when the sample was subjected to the $100 \%$ power XFEL pulses. Delocalized, random ionization events cannot account for the observed forbidden reflections.

Figure 3 shows the appearance of these forbidden reflections, coinciding with a substantial reduction in the intensities of the allowed FCC reflections. These changes cannot be described by any specific orientational ordering of ideal $\mathrm{C}_{60}$ molecules in the crystal lattice.

According to our analysis ${ }^{1}$, a correlated, non-centrosymmetric charge distribution on each $\mathrm{C}_{60}$ molecule (Eq. 4), has proved the only means of generating a model powder diffraction profile which matches the experimental data (seen in Figure 5). For comparison, all data and models are shown together, but offset vertically with respect to one another, on the same axis in Figure 6 . 


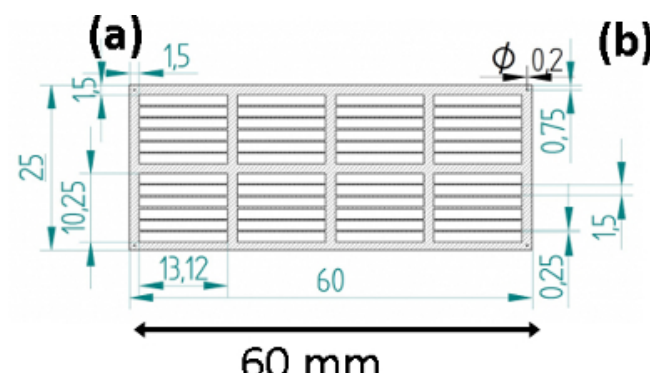

(b)

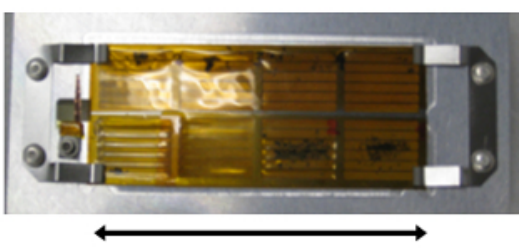

$60 \mathrm{~mm}$

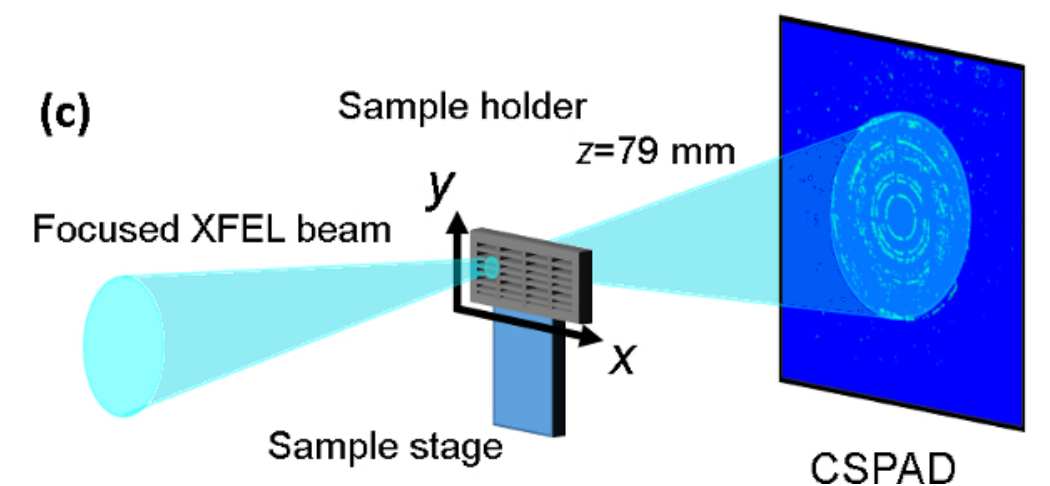

Figure 1. XFEL Powder Diffraction Sample Setup and Geometry

(a) The sample holder used for the fixed target scanning mode of $\mathrm{C}_{60}$ crystal powder. The sample frame is constructed from aluminium. Measurements indicated are in units of $\mathrm{mm}$. Approximate dimensions of sample cells are $2 \mathrm{~mm} \times 12 \mathrm{~mm}$. (b) Photograph of $\mathrm{C}_{60} \mathrm{crystal}$ powder applied in three of the cells (seen as darkly coloured cells) with polyimide backing applied as a support (the yellow film on top of the sample holder). (c) Schematic of the $\mathrm{C}_{60}$ experiment. The sample is raster scanned in $\mathrm{x}-\mathrm{y}$ directions in the snapshot imaging scheme. K-B mirrors focus the XFEL beam to a spot size of $300 \mathrm{~nm} \times 300 \mathrm{~nm}$ at the sample. Samples are held in vacuum to stabilize the sample conditions and minimize the possibility of X-ray interaction with scattering sources other than the sample. Incoming XFEL pulses hit the crystal powder held in the sample holder cells, and a diffraction pattern is recorded at the CSPAD detector. A resolution of $1.5 \AA$ is achieved by setting the sample to detector distance to $79 \mathrm{~mm}$. Please click here to view a larger version of this figure.

(a)

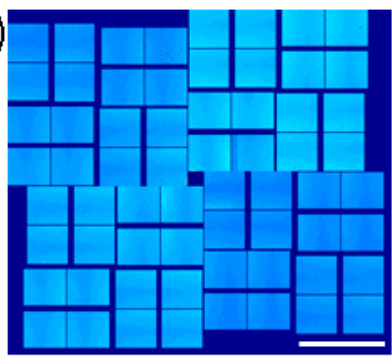

(c)

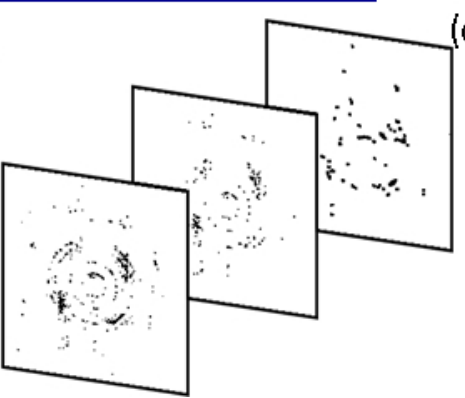

(b)

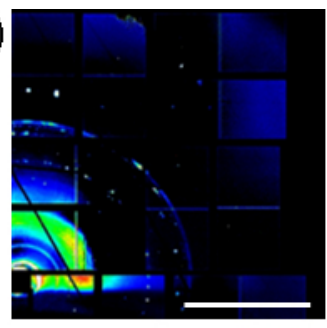

(d)

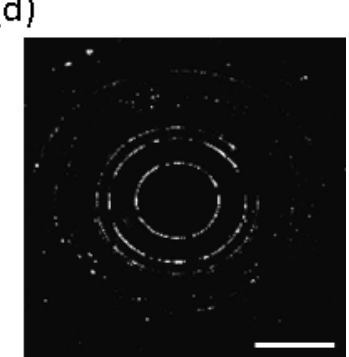

Figure 2. The CSPAD

Note that the white scale bar in a), b) and d) represents $40 \mathrm{~mm}$.

(a) CSPAD darkfield. The detector is composed of 32 rectangular modules, the positions of which can be altered by moving concentrically outward to record high-angle diffraction as needed. (b) Summed raw data frames (top right-hand quadrant, over 1000 frames summed) prior to background and darkfield correction. (c) Individual diffraction snapshots demonstrating sparsity of the diffraction signal. (d) Diffraction profile showing well defined powder diffraction rings performed by summing 1500 diffraction frames with background signal subtraction applied to individual frames. 
(a)

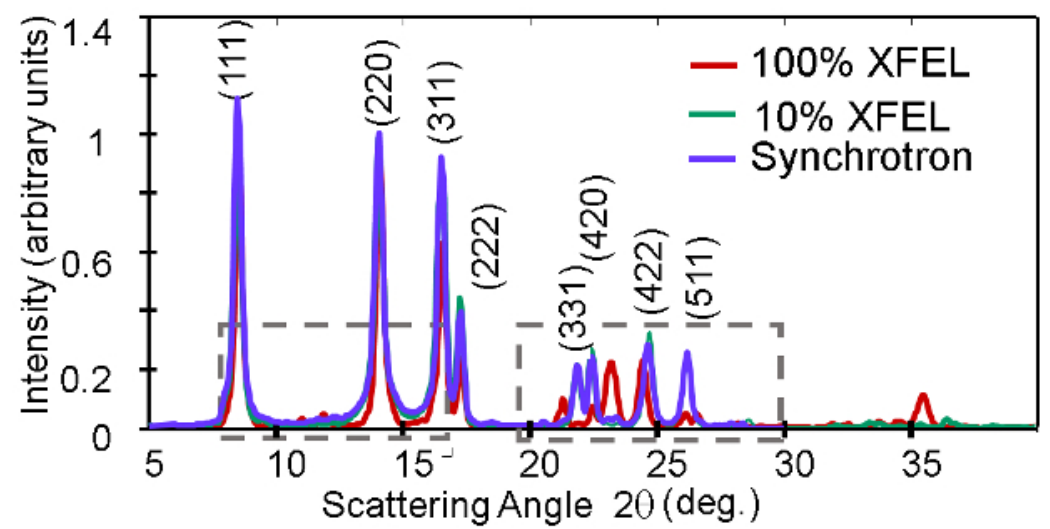

(b)

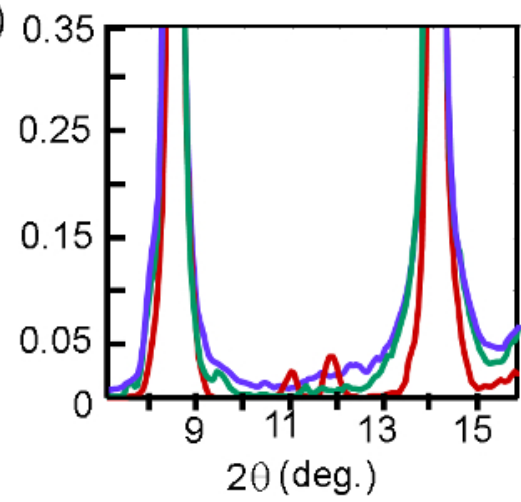

(c)

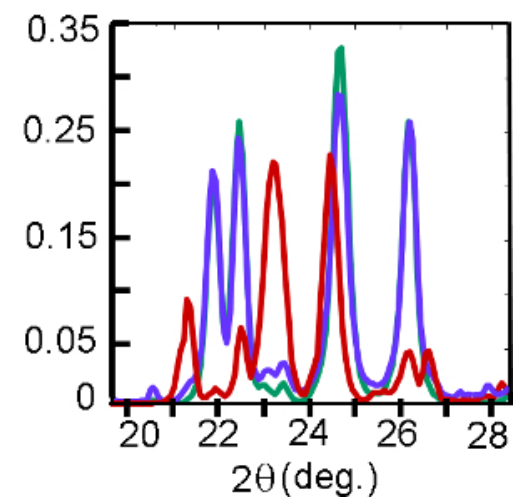

Figure 3. Powder Diffraction Data

(a) Azimuthally averaged diffraction patterns for the 10\% XFEL dataset, 100\% XFEL dataset and the Synchrotron dataset. Positions of FCC Bragg peaks are indicated consistent with a room temperature $\mathrm{C}_{60} \mathrm{FCC}$ structure. (b) Inset region showing reflections present in the $100 \% \mathrm{FCC}$ structure between scattering angles $10 \# \leq 2 \theta \leq 13 \#$ not seen in the other two profiles. (c) Inset region showing the different peak profile in the $100 \%$ XFEL data between the scattering angles $20 \# \leq 2 \theta \leq 28 \#$. The $10 \%$ XFEL data and the synchrotron data both satisfy the selection rules for FCC structures composed of electronically centrosymmetric molecules. However the presence of extra peaks (reflections) seen in the $100 \%$ XFEL data violate these selection rules. Please click here to view a larger version of this figure. 


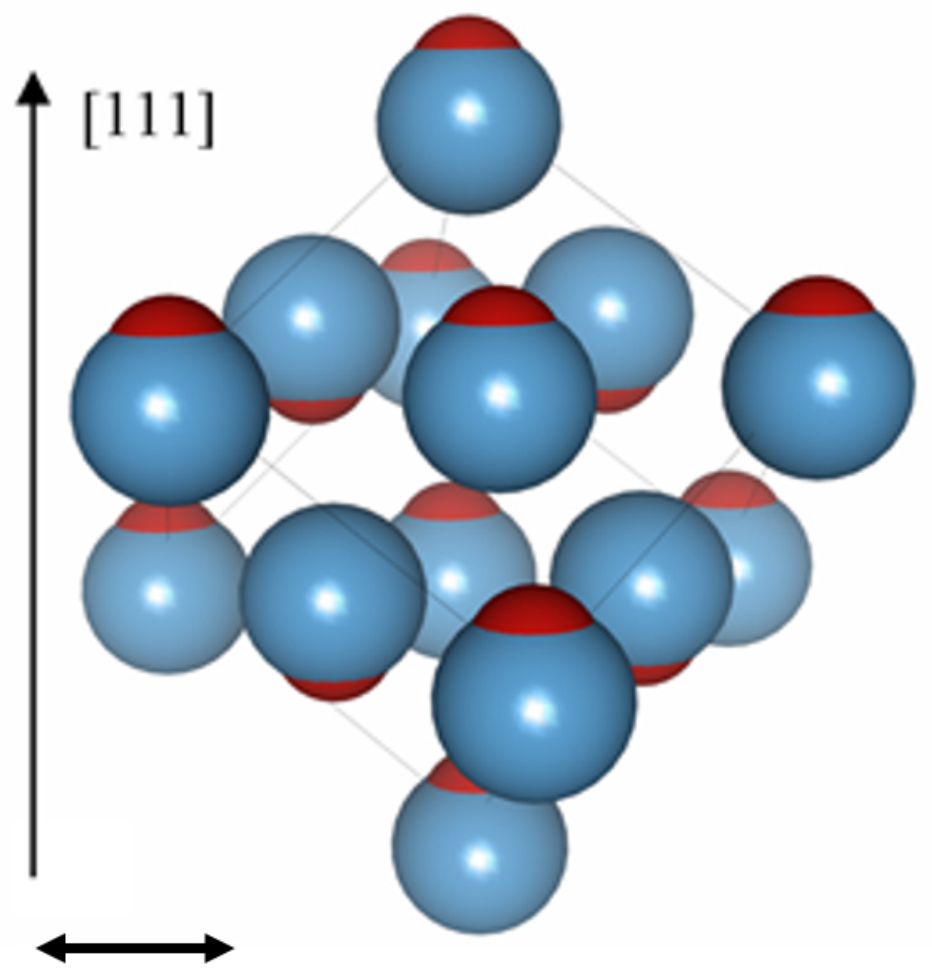

\section{$0.7 \AA$}

Figure 4. Transient Distortion of $\mathrm{C60}$

Visualisation of the alignment of the dipoles within the FCC lattice structure during the correlated electronic transient stage. $\mathrm{C}_{60}$ molecules are represented by blue spheres and the red tips represent the direction of the ordered dipoles.

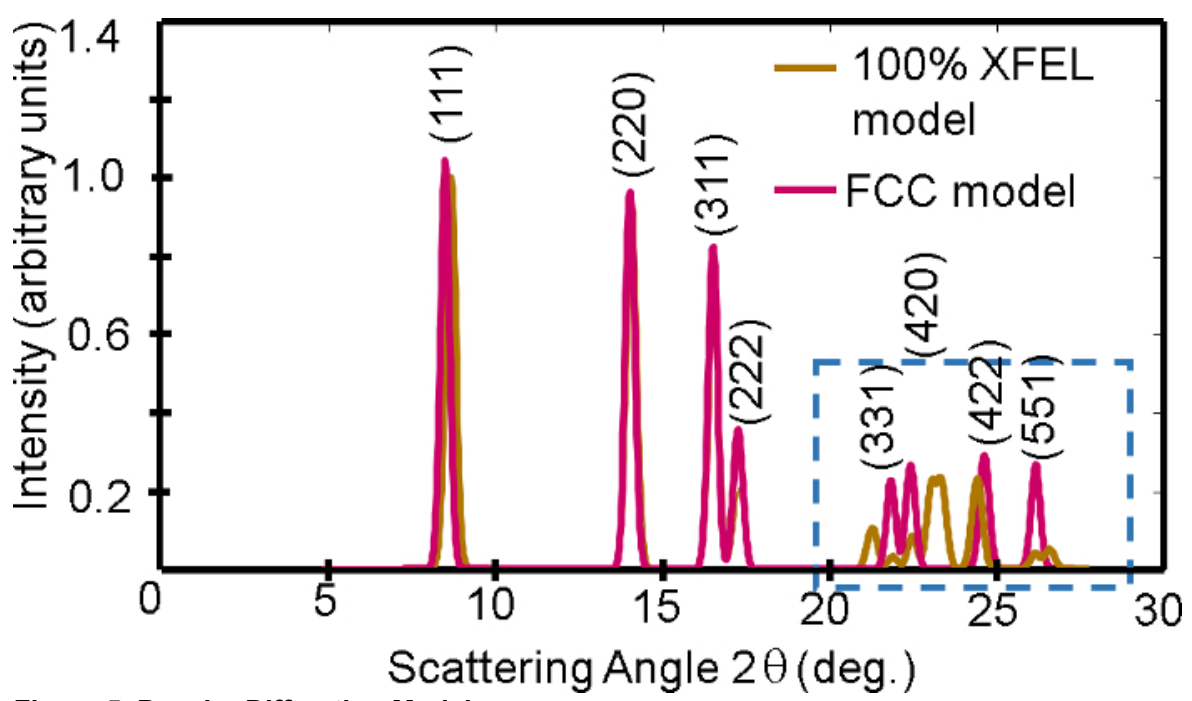

\section{Figure 5. Powder Diffraction Model}

Powder diffraction profile generated by modelling the FCC structure for $\mathrm{C}_{60}$ (using Eq 1 and 2) compared to a model of the $\mathrm{C}_{60}$ FCC structure subjected to a $100 \%$ intensity XFEL pulse (using Eq 1 and 3). Identified Bragg peaks are labelled. A region of interest $\left(20^{\circ} \leq 2 \theta \leq 30^{\circ}\right.$ ) is highlighted by the dotted line. Although the FCC model describes the intensity of the allowed reflections well, it does not explain the presence of a number of additional peaks (see Figure $2 a$ and b) observed for the $100 \%$ intensity XFEL data. The reason for this is that the simple translation of the molecular cluster (Figure 3) along the crystallographic axis of the cubic lattice gives us an incomplete picture of the orientational ordering of polarized $\mathrm{C}_{60}$ molecules in the cubic lattice. By contrast the $100 \%$ XFEL model, which takes into account ionisation-induced alignment of the dipoles within the FCC lattice (as shown in Figure 4), reproduces all of the additional peaks observed in the 100\% intensity XFEL data. Please click here to view a larger version of this figure. 

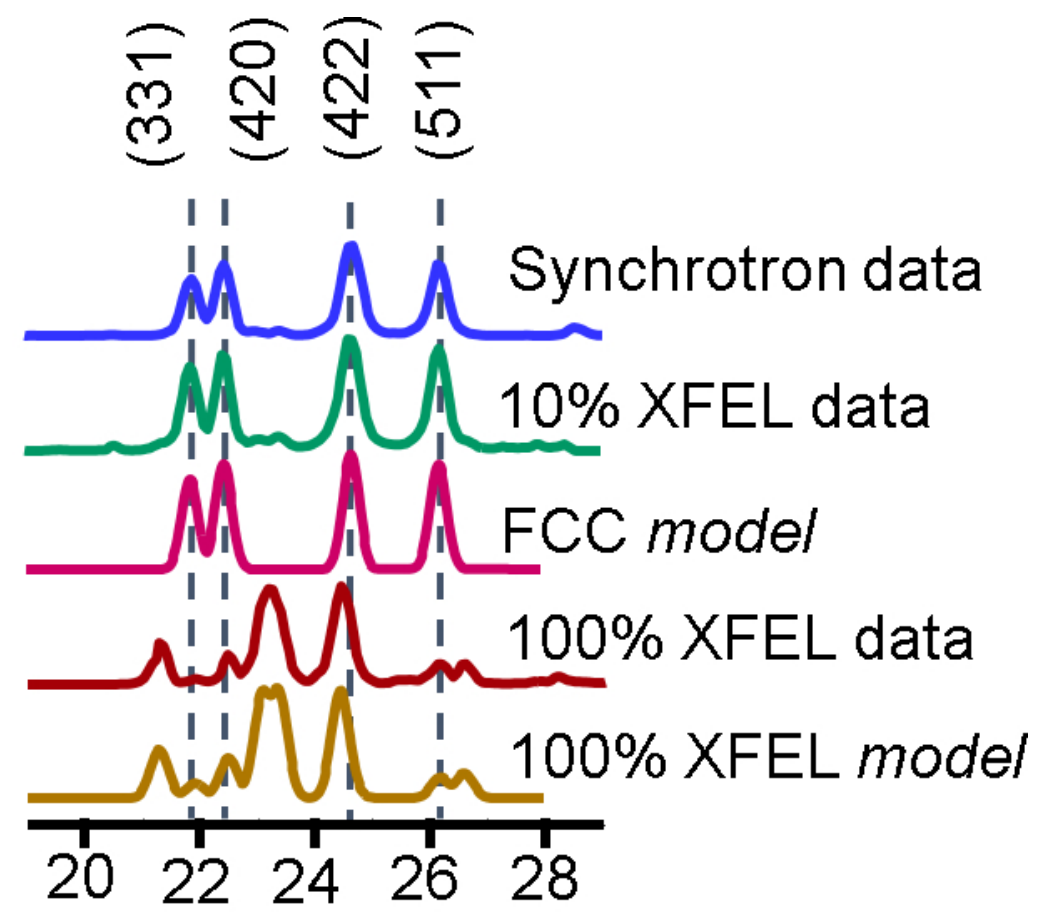

\section{Scattering Angle $2 \theta$ (deg.)}

Figure 6. Powder Profile Comparison Between Model and Data

A qualitative comparison of the line profiles for the three diffraction patterns recorded under different illumination conditions experimentally. In addition, the line profiles calculated using Equations 2 and 3 using our model are shown. It is clear that the introduction of a periodically modified MFF, the $100 \%$ XFEL model line profile agrees with our $100 \%$ XFEL data.

\begin{tabular}{|l|l|}
\hline Measured scattering angles of extra reflections (deg.) & Calculated scattering angles of extra reflections (deg.) \\
\hline 21.31 & $21.25,21.45$ \\
\hline 23.23 & $22.99,23.02,23.39$ \\
\hline 24.44 & $24.29,24.43,24.47,24.64$ \\
\hline 26.6 & $26.51,26.67$ \\
\hline
\end{tabular}

Table 1. Bragg Reflections Seen in XFEL Data

The set of Bragg reflections measured within the $20 \# \leq 2 \theta \leq 30 \#$ for the $100 \%$ XFEL diffraction data as well as those calculated using Eqns. 1 4.

\begin{tabular}{|l|l|}
\hline Position of the molecule & Alignment \\
\hline$(0,0,0)$ & $\delta r \|[\mathbf{1 1 1}]$ \\
\hline$(0.5,0.5,0)$ & $\delta r \|[\mathbf{1 1 1}]$ \\
\hline$(0.5,0,0.5)$ & $\delta r \|[\overline{\mathbf{1}} \overline{\mathbf{1}} \overline{\mathbf{1}}]$ \\
\hline$(0,0.5,0.5)$ & $\delta r \|[\overline{\mathbf{1}} \overline{\mathbf{1}} \overline{\mathbf{1}}]$ \\
\hline
\end{tabular}

Table 2. FCC Molecular Alignment During Transient Correlated Phase

This table describes the alignment of polarized $\mathrm{C}_{60}$ molecules during the transient correlated phase of the crystal experienced during the XFEL pulse.

\section{Discussion}

\section{Calibration of diffraction data frames.}

The XTC files (which contain the data from a complete run) contain calibration parameters that define the geometrical arrangement of CSPAD modules (shown in Figure 2a) during the experiment. The correct arrangement of data recorded on individual modules is crucial to assemble the individual diffraction data images comprising data recorded in each run. At the time the experiment was performed the location of the calibration file containing the correct parameters was not automatically set up and manual computation was required by the team to correct the issue. Due 
to the extra time spent performing calibration of the data there was a time-lag between setting a snapshot run dataset and checking the success of the run via a darkfield and background subtracted summation of image frames in the data set.

\section{Crystal sizes.}

In some of the initial XFEL snapshot runs, strong single crystal Bragg reflections were seen in some of the image frames. This resulted from some of the $\mathrm{C}_{60}$ sample not being crushed finely enough. Observing optical reflections from crushed powder indicates that the crystal facets are too large (correspond to the wavelength of visible light $\sim 400-700 \mathrm{~nm}$ ). The powder should be checked for these reflections at the crushing stage, and if strong, single crystal Bragg reflections are seen in the data the powder needs to be further crushed.

Since the results of this experiment were not expected or planned for successful powder diffraction data collection for the $\mathrm{C}_{60}$ sample was only obtained at two extreme intensity settings ( $10 \%$ and $100 \%$ flux). Beam time at the facility is limited and hence any set-up, computation, or sample processing errors and issues have a large impact on an experimental plan. The two most widely separated incident intensity points were prioritized and there was insufficient beam time available to collected reliable statistics for any intermediate points. Therefore, we were not able to experimentally assess the trigger point in terms of XFEL flux at which this transient phase change occurs.

\section{Preliminary studies.}

Collecting powder diffraction data at the Australian Synchrotron, from the same $\mathrm{C}_{60}$ sample as measured at the XFEL. Synchrotrons are routinely used to screen for suitable XFEL targets ${ }^{26}$, and in the present case positively confirmed that at $10 \%$ XFEL intensity, the diffraction data was consistent with the ground state FCC structure of $\mathrm{C}_{60}$.

\section{Sample and detector attenuation.}

Calibration of the incident flux through adjustment of the silicon attenuators upstream of the sample was essential, especially since the effect being studied was intensity dependent. Construction of a suitable aluminium attenuator at the detector, matched to the incident flux was also critical.

\section{Hitting the sample at the location of the beam focal point.}

The location of the KB focal spot at the XFEL was also essential to observe the reported phenomenon, since the flux density on the sample must be sufficient to induce the formation of dipoles throughout the crystal. Measuring the size of craters created by the XFEL beam in a YAG crystal using optical microscopy, as well as performing a fine sample scan along the optical axis and looking at the diffraction intensity was used to determine the location of the focal plane.

In future implementations of this work a larger number of incident intensities as well as pulse durations will be explored. This work has potential implications for upcoming experiments analyzing the diffraction data collected from nanocrystals at XFEL sources. It also provides new insights into the fundamental interaction of XFELs with matter, highlighting that XFELs have the potential to explore new physics not accommodated within conventional crystallography.

\section{Disclosures}

The authors have nothing to disclose.

\section{Acknowledgements}

The authors acknowledge the support of the Australian Research Council Centre of Excellence in Advanced Molecular Imaging. Portions of this research were carried out at the LCLS, a national user facility operated by the Stanford University on behalf of the U.S. Department of Energy, Office of Basic Energy Sciences. We acknowledge the travel funding provided by the International Synchrotron Access Program managed by the AS and by the Australian government. In addition, some of this research was undertaken on the MX1 and MX2 beamlines at the AS, Victoria, Australia. Author contributions: B.A. was responsible for planning and managing all experimental aspects of the project. Experiments were designed by B.A., R.A.D., V.S., C.D., and G.J.W. B.A., H.M.Q., K.A.N., and R.A.D. wrote the original LCLS proposal. D.W., R.A.D., R.A.R., A.V.M., E.C., and S.W. carried out the simulation work. B.A., R.A.D., C.D., V.S., M.W.M.J., R.A.R., N.G., F.H., G.J.W., S.B., M.M., M.M.S., A.G.P., C.T.P., A.V.M., and K.A.N. collected the experimental data at the LCLS. S.W., V.A.S. and R.A.D collected experimental data at the Australian Synchrotron. C.T.P. and A.V.M. led the experimental data conversion and analysis. B.A., C.D., N.G., and E.B. were responsible for the sample holder design and testing. R.A.R, B.A., S.W., A.V.M and H.M.Q wrote this manuscript. The formulation of electronic damage within coherence theory is performed by H.M.Q. and K.A.N.; R.A.D. conceived the idea to apply this formalism to C60.

\section{References}

1. Abbey, B. et al. X-ray laser-induced electron dynamics observed by femtosecond diffraction from nanocrystals of Buckminsterfullerene. Sci. Adv. 2 (9), e1601186 (2016).

2. Chapman, H. N. et al. Femtosecond X-ray protein nanocrystallography. Nat. 470 (7332), 73-77 (2011).

3. Boutet, S. et al. High-resolution protein structure determination by serial femtosecond crystallography. Sci. 337 (6092), $362-364$ (2012)

4. Redecke, L. et al. Natively inhibited Trypanosoma brucei cathepsin B structure determined by using an X-ray laser. Sci. 339 (6116), $227-230$ (2013).

5. Kern, J. et al. Simultaneous femtosecond X-ray spectroscopy and diffraction of photosystem II at room temperature. Sci. 340 (6131), $491-495$ (2013).

6. Aquila, A. et al. Time-resolved protein nanocrystallography using an X-ray free-electron laser. Opt. Exp. 20 (3), $2706-2716$ (2012). 
7. Nass, K., \& Hau-Riege, S. Radiation damage in ferredoxin microcrystals using high intensity X-FEL beams. Lawrence Livermore National Laboratory (LLNL), Livermore, CA, (2014).

8. Quiney, H. M., \& Nugent, K. A. Biomolecular imaging and electronic damage using X-ray free-electron lasers. Nat. Phys. 7 (2), $142-146$ (2011).

9. Lorenz, U., Kabachnik, N., Weckert, E., \& Vartanyants, I. Impact of ultrafast electronic damage in single-particle x-ray imaging experiments. Phys. Rev. E. 86 (5), 051911 (2012).

10. McPhillips, T.M. et al. Blu-Ice and the Distributed Control System: software for data aquisition and instrument control at macromolecular crystallography beamlines. J. Synchrotron Rad. 9 401-406 (2002).

11. Boutet, S., \& Williams, G. J. The coherent X-ray imaging (CXI) instrument at the Linac Coherent Light Source (LCLS). New J. of Phys. 12 (3), 035024 (2010).

12. LCLS Photon Control and Data Systems Documentation Page. <https://confluence.slac.stanford.edu/display/PCDS/PCDS+Home> (2009).

13. Hart, P. et al. The CSPAD megapixel x-ray camera at LCLS. Proc. SPIE. 8504 (2012).

14. Hunter, M. S. et al. Fixed-target protein serial microcrystallography with an x-ray free electron laser. Nat. Sci. Rep. 4 6026 (2014).

15. Nakane, T., et al. Data processing pipeline for serial femtosecond crystallography at SACLA. J. App. Crystallography. 49 1035-1042 (2016).

16. White, T. A. et al. Crystallographic data processing for free-electron laser sources. Acta. Cryst. D69 1231-1240 (2013).

17. Hammersley, A., et al. Two-Dimensional Detector Software: From Real Detector to Idealised Image or Two-Theta Scan. High Pressure Res. 14 235-248 (1996).

18. Dong, C. PowderX: Windows-95-based program for powder X-ray diffraction data processing. J App. Crystallography. 32 (4), 838 (1999).

19. Multi-Purpose Pattern Fitting System REITAN-FP. Adv. Ceramics Research Cnt. Nagoya Institute of Technology. (2014).

20. Ida, T., M. Ando, H. Toraya. Extended pseudo-Voigt function for approximating the Voigt profile. J. App. Crystallography. 33 (6), 1311-1316 (2000).

21. Toraya, H. Array-type universal profile function for powder pattern fitting. Journal of Applied Crystallography. 23 485-491 (1990).

22. Takata, E. N., Sakata, M. Charge density studies utilizing powder diffraction and MEM. Exploring of high Tc superconductors, $\mathrm{C}_{60}$ superconductors and manganites. Cryst. Mat. 216 (2), 2194-4946 (2009).

23. Neutze, R., Wouts, R., van der Spoel, D., Weckert, E., \& Hajdu, J. Potential for biomolecular imaging with femtosecond X-ray pulses. Nat. 406 (6797), 752-757 (2000).

24. Hau-Riege, S. P., London, R. A., \& Szoke, A. Dynamics of biological molecules irradiated by short x-ray pulses. Phys. Rev. E. 69 (5), 051906 (2004).

25. Petersen, J. C., et. al. Clocking the Melting Transition of Charge and Lattice Order in 1T-TaS ${ }_{2}$ with Ultrafast Extreme -Ultraviolet AngleResolved Photoemission Spectroscopy. Phys. Rev. Let. 107 (17), 177402 (2011).

26. Darmanin, C. et al. Protein crystal screening and characterization for serial femtosecond nanocrystallography. Nat. Sci. Rep. 6 25345 (2016). 